\title{
Characterization of single-molecule pentanedithiol junctions by inelastic electron tunneling spectroscopy and first-principles calculations
}

\author{
Carlos R. Arroyo, ${ }^{1}$ Thomas Frederiksen, ${ }^{2}$ Gabino Rubio-Bollinger, ${ }^{1}$ Marisela Vélez, ${ }^{1,3}$ Andrés Arnau, ${ }^{2,4,5}$ \\ Daniel Sánchez-Portal, ${ }^{4,5}$ and Nicolás Agraït ${ }^{1,3}$ \\ ${ }^{1}$ Departamento de Física de la Materia Condensada, Universidad Autónoma de Madrid, 28049 Madrid, Spain \\ ${ }^{2}$ Donostia International Physics Center (DIPC), Paseo Manuel de Lardizabal 4, Donostia-San Sebastián, Spain \\ ${ }^{3}$ Instituto Madrileño de Estudios Avanzados en Nanociencia (IMDEA-Nanociencia), Campus Cantoblanco, 28049 Madrid, Spain \\ ${ }^{4}$ Centro de Física de Materiales (CFM-MPC), Centro Mixto CSIC-UPV, Apdo. 1072, Donostia-San Sebastián, Spain \\ ${ }^{5}$ Depto. de Física de Materiales, Facultad de Química, UPV/EHU, Apdo. 1072, Donostia-San Sebastián, Spain
}

(Received 18 December 2009; revised manuscript received 12 January 2010; published 4 February 2010)

\begin{abstract}
We study pentanedithiol molecular junctions formed by means of the break-junction technique with a scanning tunneling microscope at low temperatures. Using inelastic electron tunneling spectroscopy and firstprinciples calculations, the response of the junction to elastic deformation is examined. We show that this procedure makes a detailed characterization of the molecular junction possible. In particular, our results indicate that tunneling takes place through just a single molecule.
\end{abstract}

DOI: 10.1103/PhysRevB.81.075405

PACS number(s): 73.40.-c, 31.10.+z, 63.22. $-\mathrm{m}, 73.63 . \mathrm{Rt}$

One of the most challenging aspects to reach ultimate miniaturization of electronic devices is the characterization of charge transport through individual molecules in contact with metallic electrodes. ${ }^{1}$ However, from an experimental point of view, it is very difficult to ensure that the junction consists of just a single molecule, and-even when such junctions are realized-it is hard to know the microscopic arrangement, e.g., how the molecule is bound to the electrodes or the pathway followed by the electrons in the molecule. A powerful tool for understanding electron transport in nanoscopic devices is inelastic electron tunneling spectroscopy (IETS). ${ }^{2-4}$ This technique is based on the variations in the conductance caused by the excitation of vibrations by the traversing electrons. Some of the characteristic molecular vibrations have been identified in junctions comprising a relatively large number of molecules in self-assembled monolayers of alkanedithiol and other thiolated molecules. ${ }^{5-7}$ In junctions of short molecules, such as water or benzene, weakly bound to the electrodes, inelastic spectroscopy has been used to explore the molecular conformations in a single-molecule junction. ${ }^{8}$ Recently, Hihath et al. ${ }^{9}$ measured IETS spectra of junctions with short alkanedithiols (propanedithiol) and showed that the average of spectra taken on the same conductance plateau were sensitive to changes in the configuration of the junction as a whole and were associated with a plastic deformation of the junction. However, the observed changes permitted neither to ensure the presence of a single molecule at the junction nor to discern its bonding configuration and state of strain, something that requires a detailed comparison between individual spectra and first-principles calculations of the inelastic electronictransport process.

Here, we report the evolution of IETS in single pentanedithiol molecules covalently bound to gold electrodes as the molecular junction is deformed elastically, i.e., with no change in molecular configuration. The measurements are performed using a scanning tunneling microscope (STM) at low temperatures $(4.2 \mathrm{~K})$ and compared to first-principles calculations, which permits a unique identification of the main peaks in the vibrational spectra, as well as their variation with strain. This further allows us to conclude unambiguously that electrons tunnel through just a single molecule suspended between the electrodes.

Single-molecule junctions are obtained by repeatedly forming and breaking the contact between an STM tip and a gold substrate covered with pentanedithiol molecules. The molecules were deposited on the substrate by immersion in a $1 \mathrm{mM}$ solution of pentanedithiol molecules in toluene for 12 $\mathrm{h}$ followed by washing and sonication in pure toluene, and drying in a stream of helium gas. Immediately after that, the sample was transferred to the low-temperature insert in helium gas atmosphere. Before immersion the substrate was treated with a piranha solution and flame annealed. The tip consisted of a freshly cut $99.99 \%$ purity gold wire. These deposition conditions are typical for the formation of selfassembled monolayers and consequently we would expect a coverage close to a monolayer. During the process of contact formation and breaking the conductance of the junction is measured by recording the current at a fixed bias voltage, of typically $25 \mathrm{mV}$, following a procedure similar to the one used for the study of $\mathrm{Au}$ atomic contacts, ${ }^{10}$ single-atom chains, ${ }^{11,12}$ and single-propanedithiol junctions. ${ }^{9}$

After rupture of a clean gold contact at low temperatures the current at a constant tip-substrate bias voltage decreases exponentially with a high apparent tunneling barrier of 3-4 $\mathrm{eV}$, which is characteristic of vacuum tunneling. ${ }^{13}$ In contrast, the presence of the molecules on the substrate gives rise to plateaus in the conductance, as shown in the inset of Fig. 1(a). The lowest conductance plateau before rupture is associated with a single molecule suspended between substrate and tip in a stable configuration. ${ }^{14}$ Consistently with the deposition conditions, the initial distribution of molecules on the substrate is quite homogeneous. However, repeated junction formation on the same spot resulted in a depletion of molecules. When one of these low-conductance plateaus is detected, tip retraction is stopped, and the current $I$, and differential conductance, $d I / d V$, are measured as a function of the applied bias voltage $V$. The differential con- 

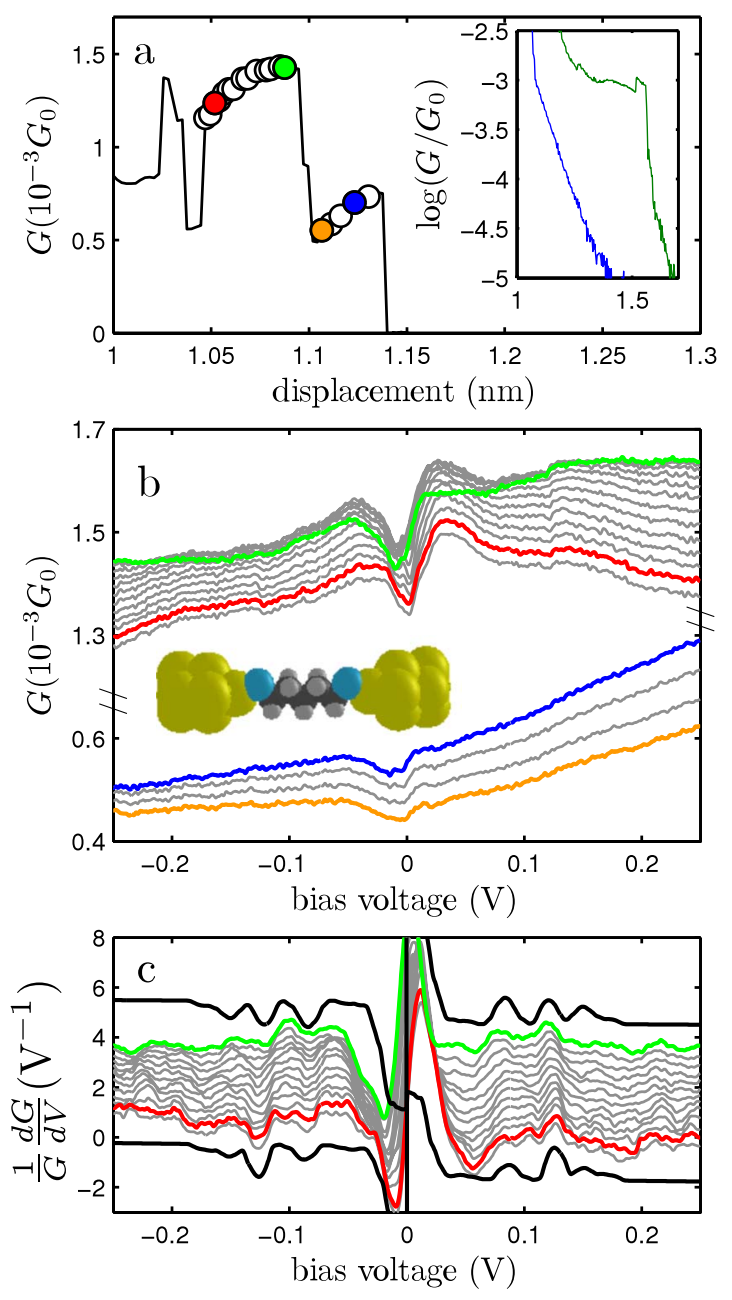

FIG. 1. (Color online) (a) Selected conductance trace corresponding to the formation of a stable molecular junction. Note the jump in conductance at a displacement of about $1.1 \mathrm{~nm}$, caused by the strain relaxation in the junction. (Inset) Low-voltage conductance traces during electrode separation after breaking a gold contact for a clean metal junction (blue/lower trace) and for a junction in which a molecule is present (green/upper trace). (b) Differential conductance vs voltage acquired at the positions indicated by the circles in panel (a). (c) Derivative of the differential conductance (normalized by the conductance), $G^{-1} d^{2} I / d V^{2}$, of the curves in panel (b). For clarity, the experimental curves have been shifted upwards as the molecular junction is stretched. The black thick curves are calculated spectra for two different electrode separations that differ by $0.1 \mathrm{~nm}$ (top curve corresponds to a more stretched configuration than bottom curve).

ductance is measured using a lock-in technique with an ac voltage modulation of $V_{\mathrm{rms}}=5 \mathrm{mV}$. The measurement is repeated in small incremental steps of tip retraction, leading to the acquisition of data such as those summarized in Fig. 1. The continuous parts of the conductance trace indicate regions where the junction deforms elastically while the sudden jumps in the conductance result from the relaxation of the accumulated stress in the junction. Most likely these relaxations are due to atomic rearrangements at the electrodes in the immediate proximity of the junction, as demonstrated in atomic wires of gold. ${ }^{15}$
The differential conductance curves, as those shown in Fig. 1(b), show a continuous evolution within a conductance plateau while sudden changes in the overall shape of the curves are observed at the conductance jumps. As in the case of atomic contacts, ${ }^{16,17}$ the large-scale features of the differential conductance curves do not reflect the properties of the junction itself but are a consequence of elastic scattering of the electrons in the vicinity of the junction and vary markedly from junction to junction. Often the differential conductance curves show a dip at low voltages. These so-called zero-bias anomalies are poorly understood and typical of low-temperature junctions. We will not give them further consideration in this work.

Careful inspection of the differential conductance curves in Fig. 1(b) shows small conductance jumps on the order of $1 \%$ at certain voltages, the most prominent of which occurs at $\pm 120 \mathrm{mV}$. These sudden changes in conductance are known signatures of inelastic scattering in the junction. ${ }^{12} \mathrm{At}$ low temperatures the vibrational motion of the molecule, which are almost completely frozen, can only be excited by the passing electrons provided the applied bias exceeds the quantum of a given vibration. In the low-transmission, offresonance case this onset of inelastic scattering leads to enhanced conductance, ${ }^{18,19}$ and hence to peaks (dips) in $d^{2} I / d V^{2}$ at positive (negative) bias polarity. Figure $1(\mathrm{c})$ shows the IETS spectra, calculated by taking the numerical derivative of the measured differential conductance curves in Fig. 1(b). Indeed, we observe antisymmetric features expected for the inelastic scattering processes.

We have studied 33 different stable molecular junctions characterized by a well-defined conductance plateau at low conductance, obtaining a total of 540 IETS spectra from the measured differential conductance curves. The probability density function (PDF) of the low-bias conductance, plotted in Fig. 2(a), shows that most of the mechanically stable junctions have a conductance of approximately $10^{-3} G_{0}$. This value is consistent with the conductance peak in the PDF observed at room temperature in solution shown in Fig. 2(b) and supports the idea that transport through these molecular junctions takes place via tunneling. Note that these two PDFs correspond to two different situations and are not directly comparable: in Fig. 2(b), the PDF is obtained from all measured traces and includes all points of the trace while in Fig. 2(a) the PDF includes a single point per spectra. We have chosen to use probability density (PDF) instead of the conventional histograms so that the probability of finding a value in a certain range can be obtained by integration of the PDF in that range. To obtain the PDF, $f(x)$ from a set of experimental data, $x_{i}$ we use the standard definition, $f(x)$ $=(1 / N) \sum_{i} \delta\left(x-x_{i}\right)$, where $N$ is the number of data and we approximate the Dirac delta function to a Lorentzian function, $\delta(x) \approx \frac{\epsilon / \pi}{x^{2}+\epsilon^{2}}$ with an adequately small $\epsilon$.

In Fig. 2(c), we show the PDF of peaks (dips) in the IETS spectra appearing at positive (negative) voltages. All peaks above the noise level are used to construct the PDF without further selection. We have normalized the PDF to the number of spectra and consequently this function represents the probability of finding a peak in a given interval of the bias voltage. The symmetry of the features reflects the antisymmetry of the IETS spectra. Some peaks are present in all 

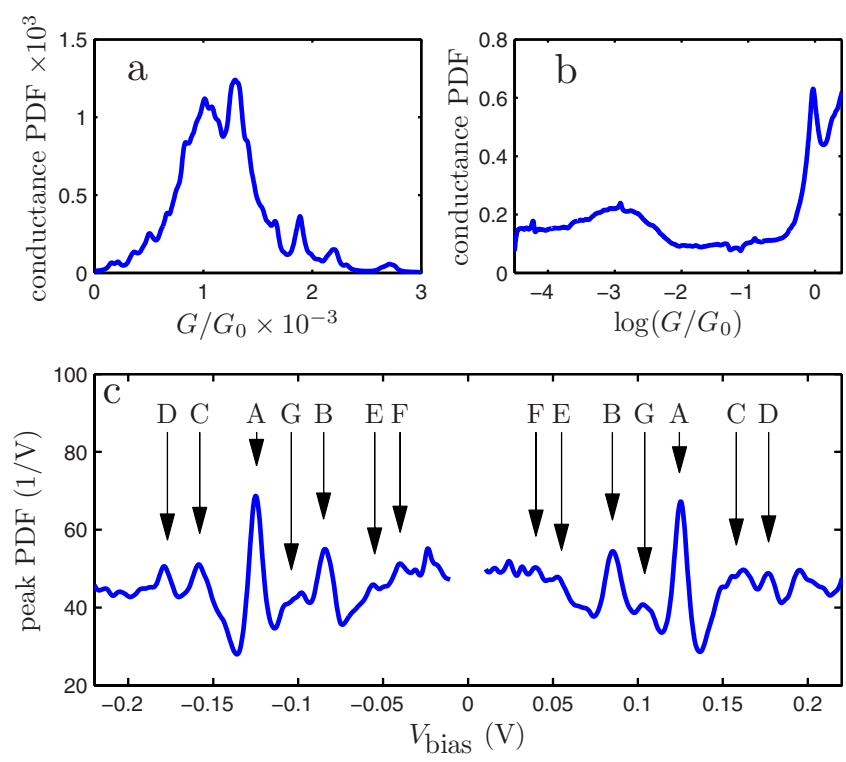

FIG. 2. (Color online) (a) PDF of the low-bias conductance for 540 spectra in 33 different molecular junctions acquired at low temperatures $(4.2 \mathrm{~K})$. The low-bias conductance of a given spectra is the ratio $I / V$ at $V=25 \mathrm{mV}$ obtained from the simultaneously measured $I$ vs $V$ curve. In the PDF we have used $\epsilon=3 \times 10^{-5}$. (b) $\mathrm{PDF}$ for the low-bias conductance measured at room temperature in solution (green curve). (c) PDF of peaks (dips) in the IETS spectra at positive (negative) voltages. Voltages around zero have been excluded due to the presence of zero-bias anomalies. In the PDF we have used $\epsilon=3 \mathrm{mV}$.

spectra. More precisely, the probability is unity for finding a peak in the interval $(125 \pm 10) \mathrm{mV}$ (peak of type A), as well as for finding one in $(85 \pm 13.5) \mathrm{mV}$ (peak of type B). These signals can be observed directly in the individual spectra, cf. Fig. 1 .

During the measurement of the IETS spectra the molecular junction is subject to a varying stress: the junction is stretched up to the breaking point. The effect of this stretching on the vibrational spectra is to shift the position of peaks of types A and B to lower voltages (a maximum of 5-10 $\mathrm{mV}$ ), i.e., to lower frequencies, as shown in Fig. 3. It is not possible to follow the evolution of the other modes as the corresponding peaks are less well defined. The observed fre-

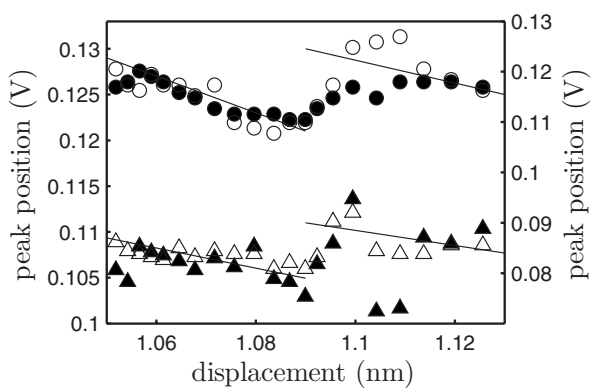

FIG. 3. Peak position as a function of the tip retraction in Fig. 1. Circles correspond to peak $\mathrm{A}$ around $125 \mathrm{mV}$ (left $y$ axis) and triangles to peak $\mathrm{B}$ around $80 \mathrm{mV}$ (right $y$ axis). Filled (open) symbols represent data obtained at positive (negative) voltage polarity. The continuous lines are a guide to the eye. quency redshift is similar to that observed for atomic gold chains, ${ }^{3}$ where the inelastic signal originates from a longitudinal mode that softens with stretching, reflecting the weakening of the interatomic bonds. ${ }^{20}$ The conductance jump at a displacement of $1.095 \mathrm{~nm}$ in Fig. 1(a) results in a frequency jump in Fig. 3, demonstrating that its origin is in a strain relaxation of the junction, probably due to an atomic rearrangement in the electrodes. ${ }^{15}$

In order to understand how the peak positions and amplitudes in the IETS spectra relate to the various vibrational modes of the molecular configuration and their dependence on stretching, we have performed first-principles transport calculations based on nonequilibrium Green's functions techniques. ${ }^{21,22}$ Our simulations attempt to mimic the experimental situation with the molecule suspended between two metal electrodes, as shown in Fig. 4, assuming that the $\mathrm{S}$ atoms are bound to $\mathrm{Au}$ adatoms on $\mathrm{Au}(111)$ surfaces. ${ }^{24}$ The calculated zero-bias conductance of the pentanedithiol junctions is of the order $0.005 G_{0}$ per molecule, i.e., about a factor of five higher than the experimental value.

Figure 4(a) shows the calculated vibrational spectrum for a pentanedithiol between two Au electrodes for two different electrode separations. The spectra are quite similar to those calculated for octanedithiol junctions. ${ }^{25}$ We observe that there are active and inactive modes due to approximate selection (propensity) rules. ${ }^{26}$ The strength of the IETS signal can be related to a matrix element involving three factors: initial electron state, vibrational deformation potential, and final electron state. Therefore, propensity rules can be understood from symmetry considerations. For the pentanedithiol junction an eigenchannel analysis shows that the IETS can be rationalized by just considering inelastic scattering between the two scattering states belonging to the most transmitting eigenchannel. These states, shown in Figs. 4(b) and 4(c) for electrons originating in the left and right electrode, respectively, possess a $\sigma$-type symmetry and can therefore only couple via modes with longitudinal character. For instance, we get a large signal from the C-C stretch mode $(\hbar \omega=127 \mathrm{meV})$ illustrated in Fig. 4(d) while scattering is suppressed for the essentially transverse mode $(\hbar \omega$ $=106 \mathrm{meV}$ ) illustrated in Fig. 4(e).

For comparison with the experimental curves the rounding introduced by the ac modulation used to measure the differential conductance has also been included in the theory curves, shown with dashed lines in Fig. 4(a) and the top and bottom curves (thick black traces) in Fig. 1(c). As a result of this rounding the individual peaks cannot be resolved. Figure 1(c) shows the excellent agreement between experiment and theory, even the magnitude of the inelastic signals is quantitatively reproduced. All the peaks appearing in the PDF in Fig. 2(c) can be identified with some group of peaks in Fig. 4(a): peak A corresponds to a C-C stretch with some contribution from $\mathrm{CH}_{2}$ twist; $\mathrm{B}$ to $\mathrm{C}-\mathrm{S}$ stretch with some $\mathrm{CH}_{2}$ rock; $\mathrm{C}$ to $\mathrm{CH}_{2}$ wag and twist; D to $\mathrm{CH}_{2}$ scissor; E to Au-S-C stretch; F to $\mathrm{Au}-\mathrm{S}$ stretch; and $\mathrm{G}$ to $\mathrm{CH}_{2}$ rock and twist. The fact that the calculated peaks have the same intensities as the measured peaks [see Fig. 1(c)] allows us to draw an important conclusion: the electrons are indeed tunneling through a pentanedithiol molecule suspended between the electrodes.

Comparison with the experiment also requires to take into 


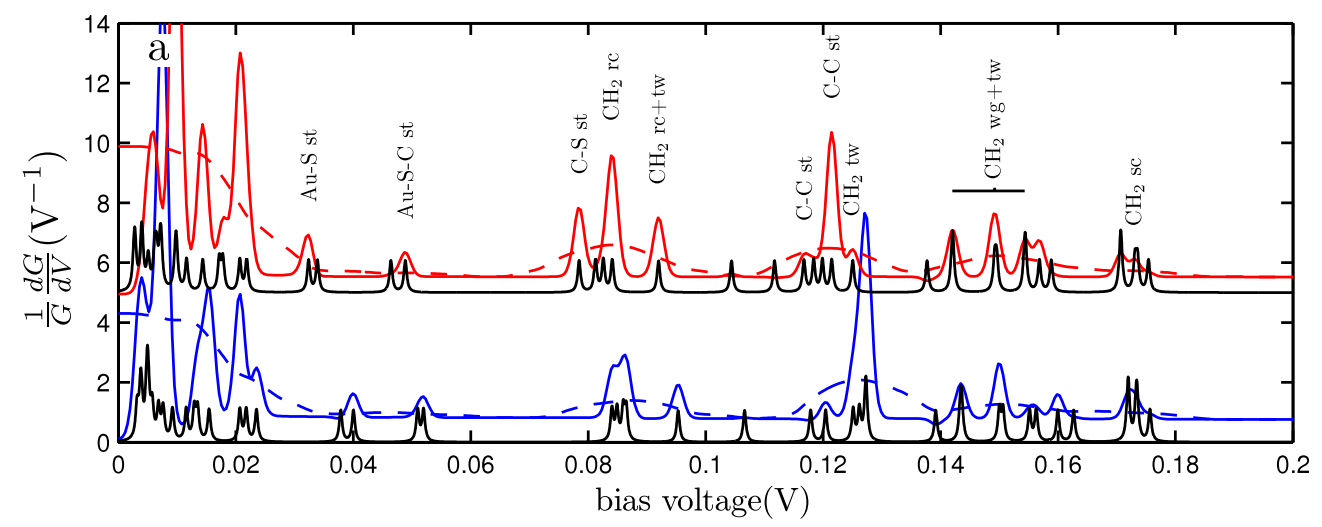

(b)

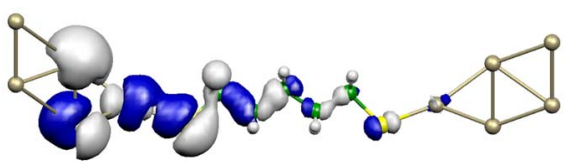

(c)

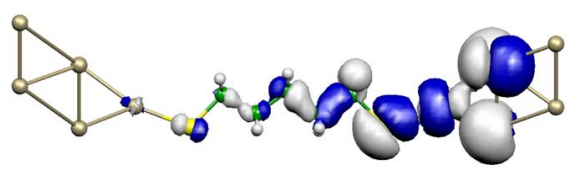

(d)

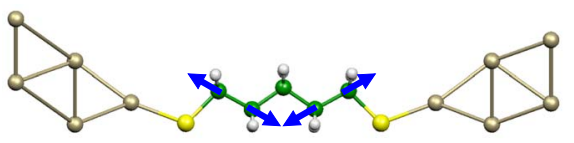

(e)

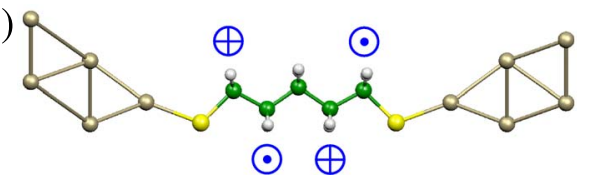

FIG. 4. (Color online) (a) Calculated IETS spectra of pentanedithiol for two different electrode separations that differ by $0.1 \mathrm{~nm}$ (top-red more stretched than bottom-blue). The dashed curves are rounded using a smoothing filter equivalent to that used in the processing of the experimental data. The corresponding black curves (vertical axis scale arbitrary) represent the density of vibrational modes for these two configurations. The different peaks are labeled according to the character of the mode $(\mathrm{st}=\mathrm{stretch}, \mathrm{rc}=\mathrm{rock}, \mathrm{wg}=\mathrm{wag}, \mathrm{tw}=\mathrm{twist}, \mathrm{sc}$ =scissor). [(b) and (c)] Eigenchannel scattering states (Ref. 23) corresponding to electrons originating in the left and right electrodes, respectively. The visualizations represent isosurfaces of the real part of the wave functions (with sign). [(d) and (e)] Illustration of an active (d) and a silent (e) mode from the spectrum for the less-stretched configuration.

consideration that the molecules are in different states of strain. We have calculated the spectra for several different elongations of the molecule to determine the strain dependence of the modes. In this way we can characterize the $\mathrm{C}-\mathrm{C}$ (C-S) mode, i.e., peak A (B), by a shift of -5.0 (-2.9) $\mathrm{meV} / \AA$, consistent with the experimental results. This is a strong indication that the junction consists of a single molecule. Indeed, if there were more molecules in the junction contributing to the transport, they would certainly be in different states of strain due to irregularities of the electrodes and, thus, preclude the observation of clear frequency shifts.

In conclusion, our results show that combining firstprinciples transport calculations and high-resolution lowtemperature IETS it is possible to characterize the configuration of single-molecule junctions. The agreement between experiment and theory is excellent and, in addition to identifying the presence of pentanedithiol molecules, allows us to extract structural information of the molecular junction, e.g., its state of strain. Indeed, the IETS signal indicates that the electrons are passing through the backbone of the molecule and that it is bound to the electrodes by the thiol groups.

T.F., D.S.P., and A.A. acknowledge stimulating discussions with Magnus Paulsson. This work has been supported by the Spanish MICINN (Grants No. MAT2008-01735, No. FIS2007-6671-C02-00, and No. MAT2007-62732, and Consolider-Ingenio 2010 under Grant No. CSD2007-0010), the CAM ("CITECNOMIK" Grant No. P-ESP-0003370505), the UPV/EHU (Grant No. IT-366-07), and the Basque Depto. de Industria and the Diputación Foral de Guipuzcoa ("ETORTEK"), the EC (STREP "SURFMOF" Grant No. NMP4-CT-2006-032109, and FP7 ITN "FUNMOLS" Grant No. 212942). T.F. acknowledges support from the Danish FNU (Grant No. 272-07-0114).
${ }^{1}$ M. Ratner, Nature (London) 435, 575 (2005).

${ }^{2}$ B. C. Stipe, M. A. Rezaei, and W. Ho, Science 280, 1732 (1998).

${ }^{3}$ N. Agraiit, C. Untiedt, G. Rubio-Bollinger, and S. Vieira, Phys. Rev. Lett. 88, 216803 (2002).

${ }^{4}$ R. H. M. Smit, Y. Noat, C. Untiedt, N. D. Lang, M. C. van
Hemert, and J. M. van Ruitenbeek, Nature (London) 419, 906 (2002).

${ }^{5}$ W. Wang, T. Lee, I. Kretzschmar, and M. A. Reed, Nano Lett. 4, 643 (2004).

${ }^{6}$ J. G. Kushmerick, J. Lazorcik, C. H. Patterson, R. Shashidhar, D. 
S. Seferos, and G. C. Bazan, Nano Lett. 4, 639 (2004).

${ }^{7}$ N. Okabayashi, Y. Konda, and T. Komeda, Phys. Rev. Lett. 100, 217801 (2008).

${ }^{8}$ M. Kiguchi, O. Tal, S. Wohlthat, F. Pauly, M. Krieger, D. Djukic, J. C. Cuevas, and J. M. van Ruitenbeek, Phys. Rev. Lett. 101, 046801 (2008).

${ }^{9}$ J. Hihath, C. R. Arroyo, G. Rubio-Bollinger, N. Tao, and N. Agraï, Nano Lett. 8, 1673 (2008).

${ }^{10}$ C. Untiedt, G. Rubio, S. Vieira, and N. Agraït, Phys. Rev. B 56, 2154 (1997)

${ }^{11}$ A. I Yanson, G. Rubio Bollinger, H. E. van den Brom, N. Agraï, and J. M. van Ruitenbeek, Nature (London) 395, 783 (1998).

${ }^{12}$ N. Agrait, A. Yeyati, and J. van Ruitenbeek, Phys. Rep. 377, 81 (2003).

${ }^{13}$ G. Rubio-Bollinger, P. Joyez, and N. Agraït, Phys. Rev. Lett. 93, 116803 (2004).

${ }^{14}$ B. Xu and N. J. Tao, Science 301, 1221 (2003).

${ }^{15}$ G. Rubio-Bollinger, S. R. Bahn, N. Agrait, K. W. Jacobsen, and S. Vieira, Phys. Rev. Lett. 87, 026101 (2001).

${ }^{16}$ B. Ludoph and J. M. van Ruitenbeek, Phys. Rev. B 61, 2273 (2000).

${ }^{17}$ C. Untiedt, G. R. Bollinger, S. Vieira, and N. Agrait, Phys. Rev. B 62, 9962 (2000).

${ }^{18}$ M. Paulsson, T. Frederiksen, and M. Brandbyge, Phys. Rev. B 72, 201101(R) (2005).

${ }^{19}$ L. de la Vega, A. Martín-Rodero, N. Agraït, and A. Levy Yeyati,
Phys. Rev. B 73, 075428 (2006).

${ }^{20}$ T. Frederiksen, M. Brandbyge, N. Lorente, and A.-P. Jauho, Phys. Rev. Lett. 93, 256601 (2004).

${ }^{21}$ M. Brandbyge, J.-L. Mozos, P. Ordejón, J. Taylor, and K. Stokbro, Phys. Rev. B 65, 165401 (2002).

${ }^{22}$ T. Frederiksen, M. Paulsson, M. Brandbyge, and A.-P. Jauho, Phys. Rev. B 75, 205413 (2007).

${ }^{23}$ M. Paulsson and M. Brandbyge, Phys. Rev. B 76, 115117 (2007).

${ }^{24}$ The electronic-structure calculations are based on the SIESTA density-functional-theory code (Ref. 27) using the GGA-PBE functional for exchange-correlation, DZP for $\mathrm{H}, \mathrm{C}$, and $\mathrm{S}$ atoms and SZP for Au, a k-point mesh of $2 \times 2 \times 2$, and 200 Ry cutoff for real-space integrations. The forces on the molecule and the $\mathrm{Au}$ adatoms were relaxed until residual forces were smaller than $0.02 \mathrm{eV} / \AA$. Intramolecular $e$-ph couplings were derived from finite differences (Ref. 22) and the IETS, calculated within the lowest order expansion (Ref. 18), were averaged over $10 \times 10 \mathbf{k}$ points.

${ }^{25}$ M. Paulsson, C. Krag, T. Frederiksen, and M. Brandbyge, Nano Lett. 9, 117 (2009).

${ }^{26}$ M. Paulsson, T. Frederiksen, H. Ueba, N. Lorente, and M. Brandbyge, Phys. Rev. Lett. 100, 226604 (2008).

${ }^{27}$ J. M. Soler, E. Artacho, J. D. Gale, A. García, J. Junquera, P. Ordejón, and D. Sánchez-Portal, J. Phys.: Condens. Matter 14, 2745 (2002). 